\title{
Small Volume Limits of 2-d Yang-Mills
}

\section{Robin Forman}

Department of Mathematics, Rice University, Houston, TX 77251, USA

Received January 24, 1992; in revised form May 22, 1992

Abstract. By examining the lattice gauge approximation we show that the small volume limit of the 2-dimensional Yang-Mills functional integral is the natural symplectic measure on the moduli space of flat connections.

\section{Introduction}

The subject of this paper is the small volume limit of the 2-dimensional Yang-Mills functional integral. By examining the lattice gauge approximation, we show that this limit is precisely the natural symplectic measure on the moduli space of flat connections. This answers affirmatively a question raised in [S2].

We begin by placing this result in context. Let $\Sigma$ be a compact orientable surface of genus $g>1, G$ a compact Lie group with finite center, and $P \rightarrow \Sigma$ a principal $G$-bundle. We suppose further that $\Sigma$ is endowed with a volume form $\varepsilon$, and $G$ is equipped with a bi-invariant Riemannian metric with total volume 1. For any connection $A$ on $P$, we associate the curvature $F_{A}$. (See [A-B] for details). We have

$$
F_{A}=f_{A} \varepsilon
$$

for some $\operatorname{ad}(P)$ valued function $f_{A}$. The Yang-Mills functional is defined by

$$
\mathrm{YM}_{\varepsilon}(A)=\int_{\Sigma}\left\|f_{\boldsymbol{A}}\right\|^{2} \varepsilon .
$$

Let $\mathscr{A}(P)$ be the affine space of connections on $P$. We are interested in the partition function

$$
Z(\Sigma, \varepsilon, k, P)=\int_{\mathscr{A}(P)} \mathscr{D} A e^{-\frac{1}{k^{2}} \mathrm{YM}_{\varepsilon}(A)},
$$

where $k^{2}$ is a coupling constant. More precisely, the object of interest is

$$
Z(\Sigma, \varepsilon, k)=\sum_{P} Z(\Sigma, \varepsilon, k, P),
$$


where the sum is over representatives $P$ of all topological equivalence classes of principal $G$ bundles. Note that if $G$ is simply connected, then all principal $G$ bundles over $\Sigma$ are trivial, so that

$$
Z(\Sigma, \varepsilon, k)=Z(\Sigma, \varepsilon, k, P)
$$

for any fixed $P$.

One difficulty is that this integral cannot converge because the measure

$$
\mathscr{D} A e^{-\frac{1}{k^{2}} \mathrm{YM}_{\varepsilon}(A)}
$$

is invariant under the infinite dimensional gauge group

$$
\mathscr{G}=\Omega^{0}(\Sigma, \operatorname{Ad} P) .
$$

Nonetheless, for the time being we follow [Wi] and examine the desired properties of $Z$. If $\phi$ is any diffeomorphism of $\Sigma$, then $\phi$ pulls back $\mathscr{A}(P)$ to $\mathscr{A}\left(\pi^{*} P\right)$, and $D A(P)$ to $D A\left(\pi^{*} P\right)$. Thus

$$
Z\left(\Sigma, \phi^{*} \varepsilon, k, \phi^{*} P\right)=Z(\Sigma, \varepsilon, k, P) .
$$

Summing over $P$, we learn that for any diffeomorphism

$$
\phi: \Sigma \rightarrow \Sigma
$$

we have

$$
Z\left(\Sigma, \phi^{*} \varepsilon, k\right)=Z(\Sigma, \varepsilon, k) .
$$

Moreover, by a Theorem of Moser [Mo], if $\varepsilon_{1}$ and $\varepsilon_{2}$ are volume forms on $\Sigma$ such that

$$
\operatorname{vol}\left(\varepsilon_{1}\right)=\operatorname{vol}\left(\varepsilon_{2}\right)
$$

(where $\left.\operatorname{vol}\left(\varepsilon_{i}\right)=\int_{\Sigma} \varepsilon_{i}\right)$ then there is a diffeomorphism $\phi$ such that

$$
\phi^{*} \varepsilon_{2}=\varepsilon_{1} .
$$

Therefore, the partition function depends only on

$$
v=\operatorname{vol}(\varepsilon)
$$

and we can denote it by

$$
Z(\Sigma, v, k) .
$$

Fix a volume form $\varepsilon_{0}$ with $\operatorname{vol}\left(\varepsilon_{0}\right)=1$. Then

$$
\mathrm{YM}_{\varepsilon_{0}}(A)=\int_{\Sigma}\left\|f_{A}\right\|^{2} \varepsilon_{0},
$$

where

$$
F_{A}=f_{A} \varepsilon_{0} .
$$

Since, for any $v \in \mathbf{R}^{+}$

$$
F_{A}=\left(\frac{f_{A}}{v}\right)\left(v \varepsilon_{0}\right)
$$


we have

$$
\mathrm{YM}_{v \varepsilon_{0}}(A)=\int_{\Sigma}\left\|\frac{f_{A}}{v}\right\|^{2}\left(v \varepsilon_{0}\right)=\frac{1}{v} \mathrm{YM}_{\varepsilon_{0}}(A)
$$

and

$$
Z(\Sigma, v, k)=\int_{\mathscr{A}} \mathscr{D} A e^{-\frac{1}{v k^{2}} \mathrm{YM}_{\varepsilon_{0}}(A)},
$$

where $\mathscr{A}$ is the disjoint union

$$
\mathscr{A}=\bigcup_{P} \mathscr{A}(P) \text {. }
$$

Thus, $Z$ depends only on $v k^{2}$. For simplicity, we will set $k=1$ and write

$$
Z(\Sigma, v)=\int_{\mathscr{A}} \mathscr{D} A e^{-\frac{1}{v} \mathrm{YM}_{\varepsilon_{0}}(A)}
$$

Let

$$
g: \mathscr{A} \rightarrow \mathbf{C}
$$

be a $\mathscr{G}$ invariant functional. For example, if $\gamma$ is an oriented loop in $\Sigma$, one can take

$$
g(A)=f\left(T_{\gamma}(A)\right)
$$

where $T_{\gamma}(A)$ is the parallel translation operator around the loop $\gamma$, and $f$ is any function on $G$ constant on every conjugacy class. Then we can consider

$$
\int_{\mathscr{A}} \mathscr{D} A \frac{1}{Z(\Sigma, v)} g(A) e^{-\frac{1}{v} \mathrm{YM}_{\varepsilon_{0}}(A)} .
$$

The measures

$$
\mathscr{D} A \frac{1}{Z(\Sigma, v)} g(A) e^{-\frac{1}{v} \mathrm{YM}_{\varepsilon_{0}}(A)}
$$

are rigorously constructed in [F2] and [S2] (in the case that $g=f\left(T_{\gamma}\right)$ for a large class of $\gamma$ ). See also [F1] and [S1] for the case $\Sigma=S^{2}$.

In this paper we examine lattice approximations to the Yang-Mills functional. Let $\mathscr{P}$ be a partition of $\Sigma$ into polygons such that each face is contractible. Let $V, E$, $F$ denote the space of vertices, edges and faces, respectively, of $\mathscr{P}$. Choose an orientation for each edge and face. We approximate the space $\mathscr{A}$ by

$$
\mathscr{A}(\mathscr{P})=\operatorname{Maps}(E, G) \text {. }
$$

For $a \in \mathscr{A}(\mathscr{P})$ (we will use a small $a$ to denote an approximate connection), $e \in E$, we think of $a(e)$ as parallel transport along $e$. In this case, the gauge group is

$$
\mathscr{G}(\mathscr{P})=\operatorname{Maps}(V, G),
$$

where $\gamma \in \mathscr{G}(\mathscr{P})$ acts on $a \in \mathscr{A}(\mathscr{P})$ by sending $a(e)$ to

$$
\gamma\left(e_{1}\right) a(e) \gamma^{-1}\left(e_{0}\right)
$$

where $e_{0}$ and $e_{1}$ are the vertices at the tail and head, respectively, of $e$. To each face $u$, we associate $\rho_{u}$, the $\varepsilon_{0}$ area of $u$, and $T_{u}$, the parallel translation around the 
boundary of $u$. Now define for each $a \in \mathscr{A}(\mathscr{P})$,

$$
e^{\mathrm{YM}_{v}(a)}=\prod_{u \in F} K\left(v \rho_{u}, T_{u}, 1\right)
$$

where

$$
K(t, x, y): \mathbf{R}_{+} \times G \times G \rightarrow \mathbf{R}
$$

is the heat kernel of $G$. (The definitions and properties of $K$ are reviewed in Sect. 1). We then consider

$$
Z(\Sigma, v, \mathscr{P})=\int_{\mathscr{A}(P)} \mathscr{D} a e^{-\mathrm{YM}_{v}(a)},
$$

where $\mathscr{D} a$ is the natural product measure on

$$
\mathscr{A}(\mathscr{P}) \cong \prod_{e \in E} G
$$

As shown in $[\mathrm{Wi}]$ if $\mathscr{P}^{\prime}$ is any refinement of $\mathscr{P}$,

$$
Z(\Sigma, v, \mathscr{P})=Z\left(\Sigma, v, \mathscr{P}^{\prime}\right)
$$

Now let

$$
g: \mathscr{A}(\mathscr{P}) \rightarrow G
$$

be any gauge invariant function. For example, if $\gamma$ is any closed loop which is a union of edges in $\mathscr{P}$, and $f$ is any function on $G$ which is constant on every conjugacy class, we can let

$$
g(a)=f\left(T_{\gamma}(a)\right)
$$

where $T_{\gamma}$ is the parallel transport operator around $\gamma$. We can then consider

$$
Z(\Sigma, v, g, \mathscr{P})=\int_{\mathscr{A}(\mathscr{P})} \mathscr{D} a g(a) e^{-\mathrm{YM}_{v}(a)}
$$

Again, if $\mathscr{P}^{\prime}$ is any refinement of $\mathscr{P}, g$ extends in a canonical way to a function on $\mathscr{A}\left(\mathscr{P}^{\prime}\right)$ and

$$
Z(\Sigma, v, g, \mathscr{P})=Z\left(\Sigma, v, g \mathscr{P}^{\prime}\right) .
$$

In [S2] (see also [Dr]) it is shown that for any $g, v$ and $\mathscr{P}$,

$$
\frac{Z(\Sigma, v, g, \mathscr{P})}{Z(\Sigma, v, \mathscr{P})}=\int_{\mathscr{A}} \mathscr{D} A \frac{1}{Z(\Sigma, v)} g(A) e^{-\mathrm{YM}_{\mathrm{v}}(A)},
$$

where the right-hand side is the infinite dimensional integral defined in [F 2] and [S2] (applied to the canonical extension of $g$ from $\mathscr{A}(\mathscr{P})$ to $\mathscr{A}$ ).

In [S2], Sengupta asks for the limit as $v \rightarrow 0$ of the measure

$$
\mathscr{D} A \frac{1}{Z(\Sigma, v)} e^{-\mathrm{YM}_{v}(A)}
$$


We answer this question by examining the lattice approximation. Witten proved in [Wi] that

$$
\lim _{v \rightarrow 0} Z(\Sigma, v, \mathscr{P})=\operatorname{vol} \mathscr{M}^{0} / \# Z(G),
$$

where $Z(G)$ is the center of $G, \mathscr{M}^{0}$ is the moduli space of irreducible flat connections on $P$, and the volume of $\mathscr{M}^{0}$ is that induced by the natural symplectic form on $\mathscr{M}^{0}$ (see $[\mathrm{A}-\mathrm{B}, \mathrm{Go}]$ and the remark at the end of this section). Witten proved (4) by independently calculating both sides (compare (2.67) and (4.72) of [Wi]). We show that this limit is true on the level of measures. That is, in this paper we prove

Theorem 1. For any gauge invariant function

$$
g: \mathscr{A}(\mathscr{P}) \rightarrow \mathbf{C}
$$

we have

$$
\lim _{v \rightarrow 0} Z(\Sigma, v, g, \mathscr{P})=\frac{1}{\# Z(G)} \int_{\mathscr{M}^{0}} g \frac{\omega^{n}}{n !},
$$

where $\omega$ is the natural symplectic form on $\mathscr{M}^{0}$, and $n=\frac{1}{2} \operatorname{dim} \mathscr{M}$.

Using (3) we learn

Corollary 2. The measure

$$
\lim _{v \rightarrow 0} \mathscr{D} A \frac{1}{Z(\Sigma, v)} e^{-\mathrm{YM}_{v}(A)}
$$

is the measure

$$
\frac{1}{\operatorname{vol} \mathscr{M}^{0}} \frac{\omega^{n}}{n !}
$$

on $\mathscr{M}^{0}$.

Remark. We are using a different normalization for the symplectic form on $\mathscr{M}^{0}$ than that in [A-B] and [Wi]. Namely, if $\alpha, \beta \in \Omega^{1}(\Sigma, \operatorname{ad} P)$ represent tangent vectors to $\mathscr{M}^{0},[\mathrm{~A}-\mathrm{B}]$ and $[\mathrm{Wi}]$ define a symplectic form $\tilde{\omega}$ on $\mathscr{M}^{0}$ by setting

$$
\tilde{\omega}(\alpha, \beta)=\frac{1}{4 \pi^{2}} \int_{\Sigma} \alpha \wedge \beta
$$

With this normalization, $\tilde{\omega}$ represents an integral cohomology class. However, we shall use the symplectic form

$$
\omega(\alpha, \beta)=\int_{\Sigma} \alpha \wedge \beta
$$

thus avoiding the spurious factors of $2 \pi$ which would otherwise appear in each formula.

\section{The Heat Kernel on $G$}

In this section we collect the necessary facts concerning the heat kernel on $G$. First, we recall the definition. Let $\Delta$ denote the Laplace-Beltrami operator acting on 
functions. Then $K(t, x, y)$ is the fundamental solution of the operator

$$
\frac{\partial}{\partial t}+\frac{1}{2} \Delta \text {. }
$$

That is, for all $t>0$,

$$
\left(\frac{\partial}{\partial t}+\frac{1}{2} \Delta_{x}\right) K(t, x, y)=0,
$$

and

$$
\lim _{t \rightarrow 0} K(t, x, y)=\delta(x-y),
$$

where $\delta(x-y)$ denotes the Dirac delta function on $G$ centered at $y$. If $\left\{f_{i}\right\}$ is a complete orthonormal set of eigenfunctions of $\Delta$ with corresponding eigenvalues $\left\{\lambda_{i}\right\}$, then

$$
K(t, x, y)=\sum_{i} e^{-t \frac{\lambda_{i}}{2}} f_{i}(x) f_{i}(y) .
$$

We require the following well-known estimates on the heat kernel on a compact Riemannian manifold $M$.

1) There are constants $C_{1}$ and $C_{2}$ such that for any $x, y \in M$ and $t$ near 0 ,

$$
K(t, x, y)<C_{1}\left(1+t^{\frac{-\operatorname{dim} M}{2}}\right) e^{-C_{2} \frac{|x-y|^{2}}{2 t}} .
$$

2) There is a neighborhood $U$ of the diagonal

$$
\Delta \subset M \times M
$$

such that for $(x, y) \in U$ and $t$ near 0

$$
K(t, x, y)=(2 \pi t)^{\frac{-\operatorname{dim} M}{2}} e^{-\frac{|x-y|^{2}}{2 t}}(1+O(t)),
$$

where $|x-y|$ denotes the Riemannian distance from $x$ to $y$.

We note that in [Wi], Witten takes a different approach, and defines

$$
e^{-\mathrm{YM}_{v}(a)}=\prod_{\text {faces } u} \Gamma\left(T_{u}, v \rho_{u}\right),
$$

where

$$
\Gamma\left(T_{u}, v \rho_{u}\right)=\sum_{\alpha}(\operatorname{dim} \alpha) \chi_{\alpha}\left(T_{u}\right) e^{-\frac{v \rho_{u} c_{2}(\alpha)}{2}} .
$$

The sum runs over all isomorphism classes of irreducible representations, $\operatorname{dim} \alpha$ is the dimension of the representation $\alpha$ and $c_{2}(\alpha)$ is the quadratic Casimir operator of the group $G$.

To see that (1) and (8) are equivalent, let $\alpha$ be an irreducible representation and let $V$ denote the representation space. Choose an inner product on $V$ such that $\alpha$ is unitary, and a basis for $V$ which is orthonormal with respect to this inner product. Then for each $g \in G, \alpha(g)$ is a $\operatorname{dim} \alpha \times \operatorname{dim} \alpha$ matrix. Denote the matrix elements by

$$
v_{i j}(g) \quad 1 \leq i, j \leq \operatorname{dim} \alpha .
$$


By the Peter-Weyl theorem, the $(\operatorname{dim} \alpha)^{2}$ functions

$$
\Phi_{i j}(g)=\sqrt{\operatorname{dim} \alpha} v_{i j}(g)
$$

are orthonormal eigenfunctions of $\Delta$, each with the same eigenvalue

$$
\lambda=c_{2}(\alpha) \text {. }
$$

Then

$$
\begin{aligned}
(\operatorname{dim} \alpha) \chi_{\alpha}(g) & =\operatorname{dim} \alpha \sum_{i} v_{i i}(g) \\
& =\sum_{i j}\left[\sqrt{\operatorname{dim} \alpha} v_{i j}(g)\right]\left[\sqrt{\operatorname{dim} \alpha} v_{i j}(1)\right] \\
& =\sum_{i, j} \Phi_{i j}(g) \Phi_{i j}(1) .
\end{aligned}
$$

It also follows from the Peter-Weyl theorem that as $\alpha$ runs through all irreducible representations, the functions

$$
\sqrt{\operatorname{dim} \alpha} v_{i j}
$$

form a complete set of normalized eigenfunctions. Therefore, substituting (9) into (8) (and then comparing with (5)) yields the desired equivalence.

\section{The Proof of Theorem 1}

(i) Restriction to Flat Connections. We call a connection $a \in \mathscr{A}(\mathscr{P})$ flat if, for every face $u$,

$$
T_{u}(a)=1,
$$

where $T_{u}(a)$ denotes the parallel transport operator around the boundary of $u$. We denote the space of flat connections by $\mathscr{F}$.

Let $N$ be any open neighborhood of $\mathscr{F}$. There is a $c>0$ such that for all $a \notin N$,

$$
\inf _{\text {faces } u}\left|T_{u}(a)-1\right|>c .
$$

Thus, using the estimate (6), there is a $c>0$ such that for small $v$,

$$
\int_{a \in \mathscr{A} \backslash N} \mathscr{D} a g(a) e^{-\mathrm{YM}_{v}(a)} \leq e^{-c / v}=O\left(v^{n}\right) \text { for all } n .
$$

Thus, up to errors which vanish to all orders in $v$, the integral $Z(\Sigma, v, g)$ can be replaced by

$$
\int_{a \in N} \mathscr{D} a g(a) e^{-\mathrm{YM}_{v}(a)}
$$

where $N$ is an arbitrary neighborhood of $\mathscr{F}$.

(ii) Reduction to Non-Singular Connections. In this section we reduce the evaluation of (10) to an integral over a neighborhood of the space of non-singular flat connections. To each connection $a \in \mathscr{A}$ and each face $u$ we associate the parallel 
transport around the boundary of $u$ so that

$$
T: \mathscr{A} \rightarrow \prod_{u} G .
$$

The flat connections are precisely

$$
\mathscr{F}=T^{-1}(1,1,1, \ldots, 1) .
$$

We denote by $\mathscr{F}^{\text {sing }}$ the set of points in $\mathscr{F}$ which are critical points of the map $T$, and

$$
\mathscr{F}^{0}=\mathscr{F} \backslash \mathscr{F}^{\text {sing }} .
$$

The set $\mathscr{F}^{0}$ is an open dense set in $\mathscr{F}$ ([Go]). It follows from the implicit function theorem that $\mathscr{F}^{0}$ is a smooth submanifold of $\mathscr{A}(\mathscr{P})$.

We express the integral (10) as a sum of 2 integrals: one over a neighborhood of $\mathscr{F}^{\text {sing }}$ and one over a neighborhood of $\mathscr{F}^{0}$. With this is mind, set

$$
B_{r}\left(\mathscr{F}^{\text {sing }}\right)=\bigcup_{a \in \mathscr{F}^{\text {sing }}} B_{r}(a)
$$

where

$$
B_{r}(a)=\{b \in \mathscr{A}|| a-b \mid<r\}
$$

and

$$
\mathscr{F}_{r}^{0}=\mathscr{F} \backslash \overline{B_{r}\left(\mathscr{F}^{\text {sing }}\right)} .
$$

Let

$$
\left(\mathscr{F}^{0}\right)^{\perp} \subseteq T_{\mathscr{F} 0 \mathscr{A}}
$$

denote the normal bundle to $\mathscr{F}^{0} \subseteq \mathscr{A}$. The exponential map takes $\left(\mathscr{F}^{0}\right)^{\perp}$ to $\mathscr{A}$. Let

$$
v \subseteq\left(\mathscr{F}^{0}\right)^{\perp}
$$

denote a neighborhood of the zero-section of $\left(\mathscr{F}^{0}\right)^{\perp}$ with the property that

$$
\exp : v \rightarrow \mathscr{A}
$$

is a diffeomorphism. For $r>0$, set

$$
v_{r}=\left.v\right|_{\mathscr{F}} ^{0}
$$

Then, for every $r>0$

$$
N_{r}=B_{2 r}\left(\mathscr{F}^{\text {sing }}\right) \cup \exp \left(v_{r}\right)
$$

is an open neighborhood of $\mathscr{F}$, and thus

$$
\begin{aligned}
Z(\Sigma, 0, g) & =\lim _{v \rightarrow 0} \int_{N_{r}} \mathscr{D} \operatorname{ag}(a) e^{-\mathrm{YM}_{v}(a)} \\
& =\lim _{v \rightarrow 0} \int_{B_{2 r}\left(\mathscr{F}^{\text {sing }}\right) \backslash \exp \left(v_{r}\right)}+\lim _{v \rightarrow 0} \int_{\exp \left(v_{r}\right)} .
\end{aligned}
$$


Now, letting $r \rightarrow 0$,

$$
\begin{aligned}
Z(\Sigma, 0, g) & =\lim _{r \rightarrow 0} \lim _{v \rightarrow 0} \int_{B_{2 r}(\mathscr{F} \operatorname{sing}) \backslash \exp \left(v_{r}\right)}+\lim _{r \rightarrow 0} \lim _{v \rightarrow 0} \int_{\exp \left(v_{r}\right)} . \\
& =\lim _{r \rightarrow 0} \lim _{v \rightarrow 0} I^{\operatorname{sing}}(\Sigma, v, g, r)+\lim _{r \rightarrow 0} \lim _{v \rightarrow 0} I^{0}(\Sigma, v, g, r) .
\end{aligned}
$$

The proof is concluded by the following two lemmas.

\section{Lemma 3.}

$$
\lim _{r \rightarrow 0} \lim _{v \rightarrow 0} I^{0}(\Sigma, v, g, r)=\frac{1}{\# Z(G)} \int_{\mathscr{M}^{0}} g \frac{w^{n}}{n !} .
$$

Lemma 4.

$$
\lim _{r \rightarrow 0} \lim _{v \rightarrow 0} I^{\text {sing }}(\Sigma, v, g, r)=0 .
$$

Before proving Lemma 3, we show that Lemma 4 follows from Lemma 3.

Proof of Lemma 4 (Assuming Lemma 3).

$$
\left|I^{\text {sing }}(\Sigma, v, g, r)\right| \leqq c I^{\text {sing }}(\Sigma, v, 1, r) .
$$

Thus, Lemma 4 follows from

$$
\lim _{r \rightarrow 0} \lim _{v \rightarrow 0} I^{\operatorname{sing}}(\Sigma, v, 1, r)=0 .
$$

Setting $g=1$ in Lemma 3

$$
\begin{aligned}
\lim _{r \rightarrow 0} \lim _{v \rightarrow 0} I^{0}(\Sigma, v, 1, r) & =\frac{1}{\# Z(G)} \int_{\mathscr{M}^{0}} 1 \frac{w^{n}}{n !} \\
& =\operatorname{Vol}\left(\mathscr{M}^{0}\right) / \# Z(G) .
\end{aligned}
$$

Thus

$$
\begin{aligned}
Z(\Sigma, 0) & =\lim _{r \rightarrow 0} \lim _{v \rightarrow 0} I^{\text {sing }}(\Sigma, v, 1, r)+\lim _{r \rightarrow 0} \lim _{v \rightarrow 0} I^{0}(\Sigma, v, 1, r) \\
& =\lim _{r \rightarrow 0} \lim _{v \rightarrow 0} I^{\text {sing }}(\Sigma, v, 1, r)+\operatorname{Vol}\left(\mathscr{M}^{0}\right) / \# Z(G) .
\end{aligned}
$$

From Witten's calculation (4),

$$
Z(\Sigma, 0)=\operatorname{Vol}\left(\mathscr{M}^{0}\right) / \# Z(G)
$$

which implies the desired conclusion.

Remark. We used Witten's calculation (4) to show that the singular flat connections do not contribute in the limit. To prove this directly from the definition of $I^{\text {sing }}$ requires information about the singular set and the behavior of $T$ near the singularities. A proof along these lines can be constructed using the results of Goldman in [Go]. In particular, Goldman proves the essential fact that all singularities of $T$ are at worst quadratic. 
(iii) Proof of Lemma 3.

$$
I^{0}(\Sigma, v, g, r)=\int_{\exp \left(v_{r}\right)} \mathscr{D} a g(a) e^{-\mathrm{YM}_{v}(a)} .
$$

Using the exponential map, we pull the integrand back to $v_{r}$. The natural volume on $v_{r}$ is the product of the volume form on $\mathscr{F}_{r}^{0}$ and the volume form on the fiber. Denote this volume form, at

by

$$
(a, b) \in \mathscr{F}_{r}^{0} \times v_{a}
$$

$$
d \operatorname{vol}_{\mathscr{F}_{r}^{\circ}}(a) d \operatorname{vol}_{v_{a}}(b) \text {. }
$$

Then, at $(a, b)$

where

$$
\exp ^{*} \mathscr{D} a=d \operatorname{vol}_{\mathscr{F}_{r}^{\circ}}(a) d \operatorname{vol}_{v_{a}}(b) h(a, b)
$$

So

$$
h(a, 0)=1 .
$$

$$
I^{0}(\Sigma, v, g, r)=\int_{a \in \mathscr{F}_{r}^{0}} d \operatorname{vol}_{\mathscr{F}_{r}^{0}} \int_{b \in v_{a}} d \operatorname{vol}_{v_{a}} g\left(\exp _{a}(b)\right) h(a, b) e^{-\mathrm{YM}_{v}\left(\exp _{a}(b)\right)}
$$

Now from (7)

$$
e^{-\mathrm{YM}_{v}\left(\exp _{a}(b)\right)}=\left[\prod_{u}\left(2 \pi v \rho_{u}\right)^{-\frac{|G|}{2}} e^{-\frac{\left|T_{u}\left(\exp _{a} b\right)-1\right|^{2}}{2 v \rho_{u}}}\right](1+O(v)),
$$

where $|G|=\operatorname{dim} G$, and

$$
\left|T_{u}\left(\exp _{a}(b)\right)-1\right|^{2}=\left|\left[d T_{u}(a)\right](b)\right|^{2}+O\left(|b|^{3}\right) .
$$

Since $\left.d T\right|_{v_{a}}$ is invertible, we have $b=\sum b_{u}$ where $\left[d T_{u}(a)\right]\left(b_{u^{\prime}}\right)=0$ if $u \neq u^{\prime}$. Then

$$
\begin{aligned}
I^{0}= & (1+O(v)) \int_{a \in \mathscr{F}_{r}^{0}} d \operatorname{vol}_{\mathscr{F}_{r}^{0}} \int_{b \in v_{a}} d \operatorname{vol}_{v_{a}} g\left(\exp _{a}(b)\right) h(a, b)(2 \pi v)^{-\frac{|G||F|}{2}} \\
& \times \prod_{u}\left(\rho_{u}\right)^{-\frac{|G|}{2}} e^{-\frac{\left|\left[d T_{u}(a)\right]\left(\frac{b_{u}}{\sqrt{v_{u}}}\right)\right|^{2}+O\left(|b|^{3}\right)}{2 v}} \\
= & (1+O(v)) \int_{a \in \mathscr{F}_{r}^{0}} d \operatorname{vol}_{\mathscr{F}_{r} 0} \int_{b \in \tilde{v}_{a}} d \operatorname{vol}_{v_{a}} g\left(\exp _{a}(\tilde{b})\right) h(a, \tilde{b})(2 \pi v)^{-\frac{|G||F|}{2}} \\
& \times \prod_{u} e^{-\frac{\left|\left[d T_{u}(a)\right]\left(b_{u}\right)\right|^{2}+O\left(|b|^{3}\right)}{2 v}} \\
= & (1+O(v)) \int_{a \in \mathscr{F}_{r}^{0}} \mathrm{dvol}_{\mathscr{F}_{r}^{0}} \int_{b \in \tilde{v}_{a}} d \operatorname{vol}_{v_{a}} g\left(\exp _{a}(\tilde{b})\right) h(a, \tilde{b})(2 \pi v)^{-\frac{|G||F|}{2}} e^{-\frac{|[d T(a)](b)|^{2}+O\left(|b|^{3}\right)}{2 v}}
\end{aligned}
$$

where $\tilde{b}=\sum \sqrt{\rho_{u}} b_{u}$ and $\tilde{v}_{a}=\left\{b \mid \tilde{b} \in v_{a}\right\}$. As $v \rightarrow 0$

$$
\begin{aligned}
& \int_{b \in \tilde{v}_{a}} d \operatorname{vol}_{v_{a}} g\left(\exp _{a}(\tilde{b})\right) h(a, \tilde{b})(2 \pi v)-\frac{|G|}{2} e^{-\frac{|[d T(a)](b)|^{2}+o\left(|b|^{3}\right)}{2 v}} \\
& \quad=g\left(\exp _{a}(0)\right) h(a, 0)\left[\operatorname{det}^{-\frac{1}{2}}(d T(a))^{*}(d T(a))\right]+o(1) \\
& \quad=g(a)\left[\left.\operatorname{det}^{-\frac{1}{2}}(d T(a))^{*}(d T(a))\right|_{v_{a}}\right]+o(1)
\end{aligned}
$$


Thus, as $v \rightarrow 0$

so that

$$
I^{0}(\Sigma, v, g, r)=\int_{\mathscr{F}_{r}^{0}} d \operatorname{vol}_{\mathscr{F}_{r}^{\circ}}\left(g(a)\left[\left.\operatorname{det}^{-\frac{1}{2}}(d T(a))^{*}(d T(a))\right|_{v_{a}}\right]+o(1)\right)
$$

$$
\lim _{r \rightarrow 0} \lim _{v \rightarrow 0} I^{0}(\Sigma, v, g, r)=\int_{\mathscr{F}^{0}} d \operatorname{vol}_{\mathscr{F}^{0}} g(a)\left[\left.\operatorname{det}^{-\frac{1}{2}}(d T(a))^{*}(d T(a))\right|_{v_{a}}\right] .
$$

Let $t_{1}, \ldots, t_{|F||G|}$ denote a basis for $(\operatorname{ker} d T(a))^{\perp}$. Then

$$
\left.\operatorname{det}^{-\frac{1}{2}}(d T(a))^{*}(d T(a))\right|_{v_{a}}=\frac{\left|t_{1} \wedge \cdots \wedge t_{|F||G|}\right|}{\left|[d T(a)]\left(t_{1}\right) \wedge \cdots \wedge[d T(a)]\left(t_{|F||G|}\right)\right|} .
$$

We must now reduce this to an integral over $\mathscr{M}^{0}$.

First, we note that $\mathscr{G}(\mathscr{P}) / Z(G)$ acts freely on $\mathscr{F}^{0}$, where $Z(G)$ is embedded in $\mathscr{G}(\mathscr{P})\left(\cong G^{|V|}\right)$ along the diagonal ([Go]). Let $s_{1}, \ldots, s_{|V||G|}$ denote a basis of the Lie algebra of $\mathscr{G}(\mathscr{P})$. Then the volume of the orbit through $a$ is

Thus, if

$$
\frac{\operatorname{Vol} G(\mathscr{P})}{\# Z(G)} \frac{\left.\mid s_{1}(a) \wedge \cdots \wedge s_{|V||G|}(a)\right)}{\left|s_{1} \wedge \cdots \wedge s_{|V||G|}\right|}
$$

$$
h: \mathscr{F}^{0} \rightarrow \mathbf{C}
$$

in any gauge invariant function, then

$$
\int_{\mathscr{F}^{0}} h d \operatorname{vol}_{\mathscr{F}^{0}}=\frac{(\operatorname{Vol} G)^{|V|}}{\# Z(G)} \int_{\mathscr{M}^{0}} h v,
$$

where, for any basis $\left\{r_{1}, \ldots, r_{k}\right\}$ of $T_{a} \mathscr{M}^{0}$,

Therefore

$$
v\left(r_{1} \wedge \cdots \wedge r_{k}\right)=\frac{\left|r_{1} \wedge \cdots \wedge r_{k} \wedge s_{1}(a) \wedge \cdots \wedge s_{|V||G|}(a)\right|}{\left|s_{1} \wedge \cdots \wedge s_{|V||G|}\right|}
$$

$$
I^{0}(\Sigma, 0, g, 0)=\frac{1}{\# Z(G)} \int_{\mathscr{M}^{0}} g \tilde{v},
$$

where, for any basis $\left\{r_{1}, \ldots, r_{k}\right\}$ of $T_{a} \mathscr{M}^{0}$,

$\tilde{v}\left(r_{1} \wedge \cdots \wedge r_{k}\right)=\frac{\left|r_{1} \wedge \cdots \wedge r_{k} \wedge s_{1}(a) \wedge \cdots \wedge s_{|V||G|}(a) \wedge t_{1} \wedge \cdots \wedge t_{|F||G|}\right|}{\left|s_{1} \wedge \cdots \wedge s_{|V||G|}\right|\left|[d T(a)]\left(t_{1}\right) \wedge \cdots \wedge[d T(a)]\left(t_{|F||G|}\right)\right|}$

(iv) Review of Reidemeister Torsion. Consider a differential complex

$$
\mathscr{C}: 0 \rightarrow C^{0} \stackrel{d}{\rightarrow} C^{1} \stackrel{d}{\rightarrow} C^{2} \rightarrow \ldots \rightarrow C^{n} \rightarrow 0
$$

where each $C^{i}$ is equipped with an inner product (as we will see, this is much more than is needed for our later purposes). First, suppose $\mathscr{C}$ is acyclic. Then, we define the torsion as follows. For each $i$, let

$$
t_{1}^{i}, \ldots, t_{m_{i}}^{i} \in C^{i}
$$


be chosen so that

$$
d t_{1}^{i}, \ldots, d t_{m_{i}}^{i}
$$

form a basis for the image of $d$ in $C^{i+1}$. Then, for each $i$,

$$
\left\{t_{j}^{i}\right\} \cup\left\{d t_{j}^{i-1}\right\}
$$

forms a basis for $C^{i}$. The torsion of $\mathscr{C}, \tau(\mathscr{C})$ is defined by

$$
\tau(\mathscr{C})=\prod_{i}\left|t_{1}^{i} \wedge \cdots \wedge t_{m_{\imath}}^{i} \wedge d t_{1}^{i-1} \wedge \cdots \wedge d t_{m_{i-1}}^{i-1}\right|^{(-1)^{i+1}} .
$$

It is easy to see that $\tau$ is independent of all choices.

If $\mathscr{C}$ is not acyclic, then $\tau(\mathscr{C})$ is not a number, but rather a norm on

$$
\frac{\prod_{i \text { odd }} \operatorname{det} H^{i}(\mathscr{C})}{\prod_{i \text { even }} \operatorname{det} H^{i}(\mathscr{C})}
$$

(where, for any vector space $V$, we write $\operatorname{det} V$ for $\Lambda^{\operatorname{dim} V} V$ ).

That is, if

$$
\left\{s_{1}^{i}, \ldots, s_{l_{i}}^{i}\right\} \subseteq \operatorname{ker} d \cap C^{i}
$$

are representatives in $C^{i}$ of a basis of $H^{i}$, then

$$
s_{1}^{i}, \ldots, s_{l_{i}}^{i}, t_{1}^{i}, \ldots, t_{m_{i}}^{i}, d t_{1}^{i-1}, \ldots, d t_{m_{i-1}}^{i-1}
$$

forms a basis for $C^{i}$ (with the $t_{j}^{i}$ chosen as in (12)). Now, for

$$
s=\frac{\prod_{i \text { odd }} s_{1}^{i} \wedge \cdots \wedge s_{l_{i}}^{i}}{\prod_{i \text { even }} s_{1}^{i} \wedge \cdots \wedge s_{l_{i}}^{i}} \in \frac{\prod_{i \text { odd }} \operatorname{det} H^{i}(\mathscr{C})}{\prod_{i \text { even }} \operatorname{det} H^{i}(\mathscr{C})}
$$

we set

$$
[\tau(\mathscr{C})](s)=\frac{\prod_{i \text { odd }}\left|s_{1}^{i} \wedge \cdots \wedge s_{l_{i}}^{i} \wedge t_{1}^{i} \wedge \cdots \wedge t_{m_{i}}^{i} \wedge d t_{1}^{i-1} \wedge \cdots \wedge d t_{m_{i-1}}^{i-1}\right|}{\prod_{i \text { even }}\left|s_{1}^{i} \wedge \cdots \wedge s_{l_{l}}^{i} \wedge t_{1}^{i} \wedge \cdots \wedge t_{m_{i}}^{i} \wedge d t_{1}^{i-1} \wedge \cdots \wedge d t_{m_{i-1}}^{i-1}\right|}
$$

Again, $\tau$ is independent of our choice of $t$ 's.

Now consider the following differential complex. Fix $a \in \mathscr{F}^{0}\left(\in G^{|E|}\right)$,

$$
\mathscr{C}_{a}: 0 \rightarrow \mathfrak{g}^{|V|} \stackrel{d^{0}}{\rightarrow} \mathfrak{g}^{|E|} \stackrel{d^{1}}{\rightarrow} \mathfrak{g}^{|F|} \rightarrow 0
$$

where, for $s \in \mathfrak{g}^{|V|}$

$$
d^{0}(s)=s(a)
$$

and, for $t \in \mathfrak{g}^{|E|}$

$$
d^{1}(t)=[d T(a)](t) .
$$

Since $T$ is invariant under the action of $G$,

$$
d^{1} \circ d^{0}=0 \text {. }
$$

For $a \in \mathscr{F}^{0}, d T(a)$ is onto, so that

$$
H^{2}\left(\mathscr{C}_{a}\right)=0 .
$$


By standard duality

$$
H^{0}\left(\mathscr{C}_{a}\right)=0 .
$$

Therefore, $\tau\left(\mathscr{C}_{a}\right)$ is a norm on $\operatorname{det} H^{1}\left(\mathscr{C}_{a}\right)$, defined by

$$
\begin{aligned}
& {\left[\tau\left(\mathscr{C}_{a}\right)\right]\left(r_{1} \wedge \cdots \wedge r_{k}\right)=} \\
& \quad \frac{\left|r_{1} \wedge \cdots \wedge r_{k} \wedge t_{1} \wedge \cdots \wedge t_{|F||G|} \wedge d^{0} s_{1} \wedge \cdots \wedge d^{0} s_{|V||G|}\right|}{\left|s_{1} \wedge \cdots \wedge s_{|V||G|}\right|\left|d^{1} t_{1} \wedge \cdots \wedge d^{1} t_{|F||G|}\right|}
\end{aligned}
$$

where: $k=\operatorname{dim} H^{1}\left(\mathscr{C}_{a}\right),\left\{r_{1}, \ldots, r_{k}\right\} \subseteq \operatorname{ker} d^{1}$ are representatives of a basis of $H^{1}\left(\mathscr{C}_{a}\right),\left\{s_{1}, \ldots, s_{|V||G|}\right\}$ is a basis of $\mathfrak{g}^{|V|}$, and $\left\{t_{1}, \ldots, t_{|F||G|}\right\}$ is a basis of $\left(\operatorname{ker} d^{1}\right)^{\perp}$.

Notice that

$$
\operatorname{ker} d^{1}[=\operatorname{ker} d T(a)] \cong T_{a} \mathscr{F}^{0}
$$

and

$$
\text { Image } d^{0}=\operatorname{span}\left\{s_{1}(a), \ldots, s_{|V||G|}(a)\right\}
$$

is the tangent space to the $G$-orbit of $a$. Therefore

$$
T_{a} \mathscr{M}^{0} \cong \frac{\operatorname{ker} d^{1}}{\text { Image } d^{0}} \cong H^{1}\left(\mathscr{C}_{a}\right) .
$$

Thus, comparing (11), (13), (14) and (15) we see

$$
I^{0}(\Sigma, 0, g, 0)=\frac{1}{\# Z(G)} \int_{a \in \mathscr{M}^{0}} g(a) \tau\left(\mathscr{C}_{a}\right) .
$$

Witten proved in ([Wi], (4.19) to (4.28)), that

$$
\tau\left(\mathscr{C}_{a}\right)=\left.\frac{\omega^{n}}{n !}\right|_{a},
$$

where $\omega$ is the natural symplectic form on $\mathscr{M}^{0}$. Therefore

$$
I^{0}(\Sigma, 0, g, 0)=\frac{1}{\# Z(G)} \int_{a \in \mathscr{M}^{0}} g(a) \frac{\omega^{n}}{n !}
$$

as desired.

Acknowledgments. The author would like to thank Ambar Sengupta for his comments on an earlier version of this manuscript, and the Regional Geometry Institute in Utah for their hospitality while much of this work was completed.

\section{References}

[A-B] Atiyah, M., Bott, R.: The Yang-Mills Equations over Riemann Surfaces. Phil. Trans. Roy. Soc. Lond. A 308, 523-615 (1982)

[Dr] Driver, B.: $\mathrm{YM}_{2}$ : Continuum Expectations Lattice Convergence, and Lassos. Commun. Math. Phys. 123, 575-616 (1989)

[F 1] Fine, D.: Quantum Yang-Mills over the Two-Sphere. Commun. Math. Phys. 134, 273 (1990) 
[F 2] Fine, D.: Quantum Yang-Mills on a Riemann Surface. Commun. Math. Phys. 140, 321-338 (1991)

[Go] Goldman, B.: The Symplectic Nature of Fundamental Groups of Surfaces. Adv. in Math. 54, 200 (1984)

[Mo] Moser, J.: On the Volume Elements on a Manifold. Trans. Am. Math. Soc. 120, 286-294 (1965)

[S1] Sengupta, A.: The Yang-Mills Measure for $S^{2}$ (to appear in J. Funct. Anal.)

[S2] Sengupta, A.: Quantum Gauge Theory on Compact Surfaces (preprint)

[Wi] Witten, E.: On Quantum Gauge Theories in Two Dimensions. Commun. Math. Phys. 141, 153-209 (1991)

Communicated by A. Jaffe 